\title{
Development of a Finite Element Model of the Thor Crash Test Dummy
}

Joseph Canha, Frank DiMasi and Yim Tang US DOT/RSPA/Volpe Center

Mark Haffner US DOT National Highway Traffic Safety Administration (NHTSA)

Tariq Shams GESAC, Inc.

Reprinted From: Biomechanics Research (SP-1494) 
The appearance of this ISSN code at the bottom of this page indicates SAE'S consent that copies of the paper may be made for personal or internal use of specific clients. This consent is given on the condition, however, that the copier pay a $\$ 7.00$ per article copy fee through the Copyright Clearance Center, Inc. Operations Center, 222 Rosewood Drive, Danvers, MA 01923 for copying beyond that permitted by Sections 107 or 108 of the U.S. Copyright Law. This consent does not extend to other kinds of copying such as copying for general distribution, for advertising or promotional purposes, for creating new collective works, or for resale.

SAE routinely stocks printed papers for a period of three years following date of publication. Direct your orders to SAE Customer Sales and Satisfaction Department.

Quantity reprint rates can be obtained from the Customer Sales and Satisfaction Department.

To request permission to reprint a technical paper or permission to use copyrighted SAE publications in other works, contact the SAE Publications Group.

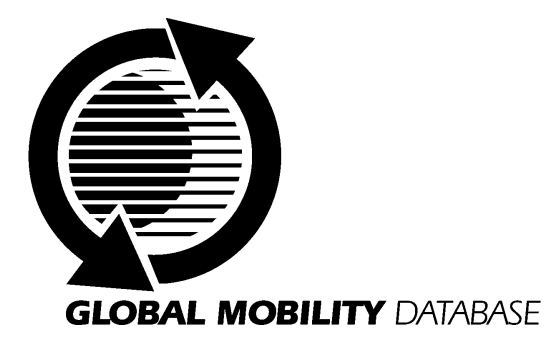

All SAE papers, standards, and selected books are abstracted and indexed in the Global Mobility Database

No part of this publication may be reproduced in any form, in an electronic retrieval system or otherwise, without the prior written permission of the publisher.

ISSN 0148-7191

Copyright 2000 Society of Automotive Engineers, Inc.

Positions and opinions advanced in this paper are those of the author(s) and not necessarily those of SAE. The author is solely responsible for the content of the paper. A process is available by which discussions will be printed with the paper if it is published in SAE Transactions. For permission to publish this paper in full or in part, contact the SAE Publications Group.

Persons wishing to submit papers to be considered for presentation or publication through SAE should send the manuscript or a 300 word abstract of a proposed manuscript to: Secretary, Engineering Meetings Board, SAE.

\section{Printed in USA}




\title{
Development of a Finite Element Model of the Thor Crash Test Dummy
}

\author{
Joseph Canha, Frank DiMasi and Yim Tang
}

US DOT/RSPA/Volpe Center

Mark Haffner

US DOT National Highway Traffic Safety Administration (NHTSA)

Tariq Shams

GESAC, Inc.

Copyright (c) 2000 Society of Automotive Engineers, Inc.

\begin{abstract}
The paper describes the development of a detailed finite element model of the new advanced frontal crash test dummy, Thor. The Volpe Center is developing the model for LS-DYNA in collaboration with GESAC, the dummy hardware developer, under the direction of the NHTSA. The modeling approach and test procedures used to create and validate each major subassembly are discussed. Specialized static and dynamic tests were performed to aid in defining and validating appropriate material models, joint characteristics, and dynamic response characteristics. The details of each dummy component are presented along with the validation procedure based on material and subassembly tests.
\end{abstract}

\section{INTRODUCTION}

The National Highway Traffic Safety Administration (NHTSA) National Transportation Biomechanics Research Center has funded and directed the development of an advanced frontal crash test dummy, named Thor, which incorporates improved biofidelic features and significantly expanded instrumentation. The primary goal of the design effort has been the creation of an effective tool for whole-body trauma assessment in a variety of automotive occupant restraint environments. The Thor dummy, expected to be released late in 1999, represents a fiftieth percentile male and has been designed and built by GESAC, Inc., of Boonsboro, MD, under the direction of the NHTSA. The finite element model presented here is intended to provide a supplemental analytical design tool, which, in combination with the physical dummy, will provide a valuable tool for crash safety research. A validated model of the Thor dummy will be a valuable tool for crash safety research.
The use, and usefulness, of vehicle/occupant crash test simulations continues to increase with enhancements in modeling and solver software as well as computing power. Although rigid body (multibody) models are less computationally expensive than finite element models, they are relatively limited in their ability to provide detailed biological and structural response data. Increasingly, finite element modeling incorporates the efficiency of rigid body modeling by using a hybrid characterization of relatively stiff (rigid) parts combined with flexible structural components, and representations of articulated connections and contact entities to provide an efficient and more detailed mechanical representation of the crash event.

Since the LS-Dyna finite element program is widely used and accepted by the automotive and biomechanics communities for crash simulation and analysis, the model is developed to run using the LS-Dyna software from LSTC (Livermore Software Technology Corporation). The modeling process is a collaborative effort between the Volpe Center and GESAC with contributions from the developers of the LS-DYNA software code, to maximize the accuracy and computational efficiency of the dummy model.

Development of both the physical and finite element dummies is sponsored and coordinated by the NHTSA. The modeling process is a collaborative effort between the Volpe Center and GESAC with contributions from the developers of the LS-DYNA software code, LSTC, to maximize the accuracy and computational efficiency of the dummy model.

\section{MODELING APPROACH}

The physical dummy is comprised of several major subassemblies and the finite element model development 
reflects that construction. The modeling effort began with the creation of an individual finite element mesh of each major part contained in each dummy subassembly. The assembled finite element mesh is shown in Figure 1. Parts that were considered to be rigid bodies were still meshed with geometric fidelity to accurately represent mass distribution and inertial properties, and to provide the option of redefining a part as a flexible body if necessary. From the beginning, the physical dummy was scrutinized to determine the most compliant components expected to have the largest effect on dummy kinematic response variables.

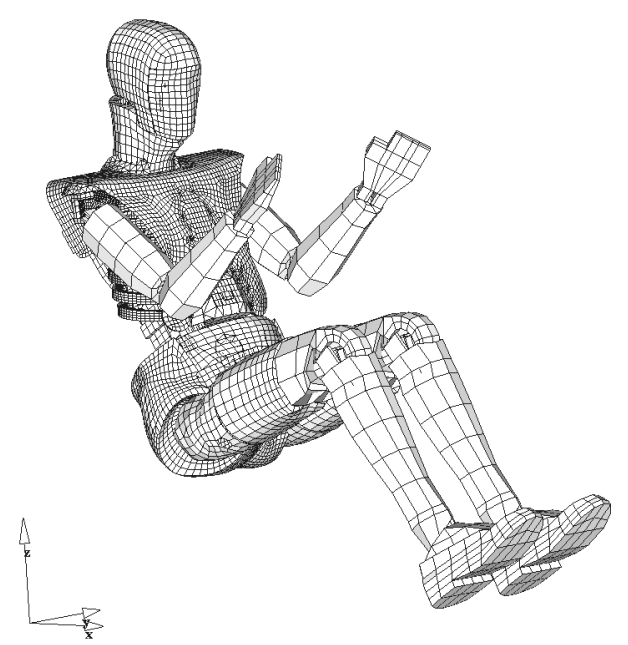

Figure 1. Assembled Thor finite element model

The geometry of the majority of parts was obtained from detailed two-dimensional AutoCAD manufacturing drawings provided by GESAC, Inc. More geometrically complex parts, such as the pelvis casting and skins, were digitized. Most geometric entities were imported into the Hypermesh pre-processor for meshing and some solid element components were meshed using the Patran mesh generation system.

The individual parts were then assembled within Hypermesh into an LS-DYNA keyword format database for each subassembly. These contained appropriate contact interactions and part connections that would be used for component test simulations.

For principal flexible entities, static and dynamic material testing [1] and finite element simulations of these tests, were performed to develop material models to best represent the material behavior. In general, for each material tested, a $50.8 \mathrm{~mm}$ cube material sample was impacted quasi-statically and at two additional impact speeds to approximate material strain rates expected in practice. These were usually between 1.6 and $3.0 \mathrm{~m} / \mathrm{s}$. Durometer measurements were also made on rubber materials.

These data were used to estimate the modulus for the elastic/plastic materials and combined static shear-com- pression tests of each specimen were also performed to tune the modulus, if necessary. The dynamic compression tests were then used to determine the material's velocity dependence and to tune material parameters under dynamic conditions.

Specialized component tests and corresponding finite element simulations of each subassembly were performed to tune material properties and to validate the dynamic response of each model subassembly. Iterative modeling of component and sub-assembly tests was conducted to tune and retune material models and construction methods to replicate the dynamic response of corresponding physical tests.

The validated subassemblies will soon be integrated into a single complete dummy database, and components were joined using joint definitions, equivalencing and/or constraint methods as appropriate. The joint properties and range of motion for each joint were obtained from quasi-static testing [2]. The properties at each limb joint were set so that they could just barely support the weight of the fully outward extended limbs, called 1-G friction.

Contact between components, or subassemblies, are included, but contact with potential external entities, such as seat belts, steering wheel, air bag, and knee bolster, will be incorporated when the model is finalized.

Although not detailed in this paper, the fully assembled dummy model will then be validated against various tests, such as the linear impactor Kroell tests, abdomen rod impacting, and whole-body head impact testing. The final stage of validation will then involve simulations with various seat belt and air bag configurations in sled or full vehicle tests.

\section{FINITE ELEMENT MODEL DETAILS}

The following section presents the details of each component including a description of the physical parts, the mesh and modeling techniques employed, and component testing performed for the creation and validation of that component. The major instrumentation of each component is also described. Except where noted, the elements of each component are eight-node solid brick elements and are modeled as rigid bodies.

HEAD - The head part consists of a metal skull casting surrounded by a vinyl skin material and attached to the neck sub-assembly at the occipital condyle. Contained within the head skull casting are nine accelerometers (three biaxial and one triaxial at the center of gravity) and two neck stop assemblies (spring, cable and housing) used to apply loads from the neck to the front and rear of the head. The new restorable load sensing face design contains five load cells and a foam pad covered in a vinyl skin. 


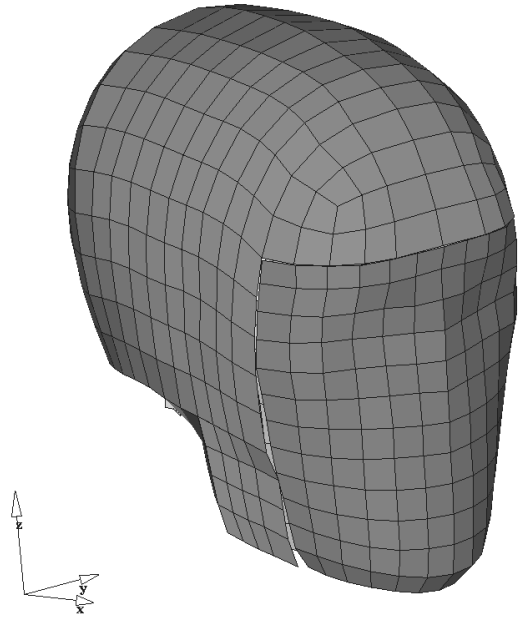

Figure 2. Head subassembly

The skull casting is modeled as a rigid body by shell elements that are given the correct inertial properties. See Figure 2 for a view of the head part. Deformable parts include the head and face skins and the face foam. The face foam is blue confor foam that is modeled with the LS-DYNA Fu-Chang Foam material model to characterize its high strain rate dependence. The skins are made of open cell foam with a vinyl covering which are modeled together as one layer of viscoelastic material. The properties of the head skin are established using standard head drop tests where the entire head is dropped from two heights. The face load cells and confor foam were dynamically tested with cylindrical rod and circular disk impacts at two speeds.

NECK - The neck structure is connected to the base of the head and, at the bottom, to the neck pitch change mechanism at the top of the spine. It consists of five elliptically shaped, neoprene rubber disks sandwiched between metal plates with a steel cable running though the center. There are two springs in the head part that connect to the neck with cables that are used to model the passive resistance of the neck in frontal flexion and extension. One cable is passed through the rear along the entire neck length and one attaches to the top front of the neck. Six axis load cells are placed at the upper and lower extremities of the neck to measure forces and torques at these locations, and a neck tilt sensor measures angular displacements of the neck. A soft foam elastic neck shroud encloses the neck hardware to prevent should belts from riding up and into the neck assembly.

Neck deformable parts include the elliptical rubber disks modeled with a viscoelastic material model, the steel cables modeled using elastic truss elements, and the neck shroud foam which is represented by a low density foam. Discrete springs are also used to model headneck resistance to flexion and extension. Figure 3 displays the mesh of the neck and the head with the neck shroud shown as transparent to reveal neck details.
The component tests performed on the neck subassembly include frontal flexion, lateral flexion, and extension bending tests performed statically and impacted at two speeds. The front and rear cables and neck shroud were not included in the tests. The test setup consisted of a rigid rod fixed to the top neck plate and impacted by another rigid rod mounted to the linear impactor. Similar testing was performed on the spine flex joints described below. It should be noted that throughout all dynamic testing, the impactor is covered with either the bib urethane or Rubatex jacket material to avoid metal to metal contact.

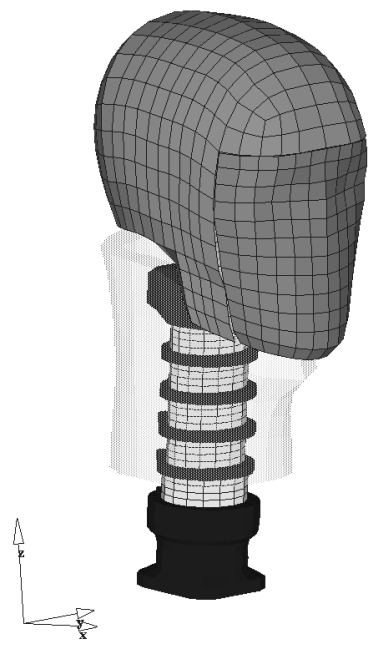

Figure 3. Neck subassembly with transparent shroud

Static compression tests on the front and rear springs were performed to extract their compression characteristics. Also, pendulum tests with the head and neck subassemblies will be performed at two speeds.

SPINE - The spine is an important structural region of the dummy and is broken up into the following subassemblies descending from the base of the neck to the top of the pelvis casting. The finite element mesh for the entire spine is shown in Figure 4. The instrumentation in the spine includes two accelerometers, four tilt (angular displacement) sensors, and a thoracic spine load cell.

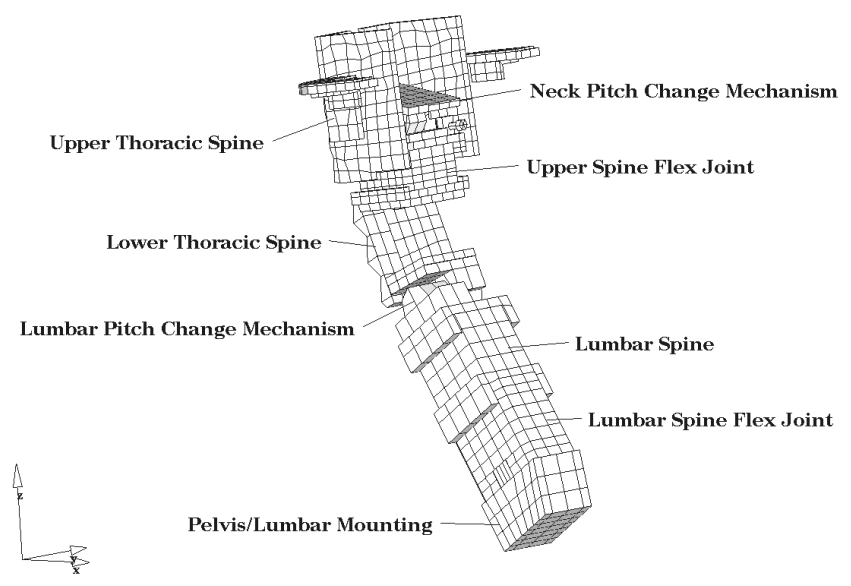

Figure 4. Spine subassemblies 
In addition to testing of the subassemblies presented here, the spine is included in the full dummy Kroell tests described later.

Neck Pitch Change Mechanism - This system connects the neck to the upper thoracic spine and is used for initial angular positioning of the head/neck assemblies with respect to the spine. The structure is modeled as rigid, and an angular displacement sensor is mounted on the top portion of the assembly.

Upper Thoracic Spine - The upper thoracic spine weldment, modeled as a rigid body, is used to attach ribs number one through four and the shoulder blocks to the spine. The top of the structure attaches to the neck via the neck pitch change mechanism and to the top of the upper spine flex joint. The chest center of gravity triaxial accelerometer is mounted on the assembly.

Upper Spine Flex Joint - This system provides slight flexibility to the upper portion of the spine and consists of a compliant molded urethane block sandwiched between two metal plates. Two short, stiff metal cables run between the metal plates to provide a fail-safe captivation device should the joint experience excessive forces. The level of biofidelity of the system, however, has not yet been quantified due to lack of dynamic data.

Each component of the system is modeled and the cables and urethane block are deformable. The cables are elastic steel beam elements and the urethane is modeled with viscoelastic bricks. The component tests performed on the flex joint subassembly include frontal flexion and lateral flexion bending tests performed statically and impacted at two speeds. Although the flex joint urethane is slightly pre-stressed by the steel cables, it is not modeled as such.

Lower Thoracic Spine - The lower thoracic spine weldment, modeled as a rigid body, is used to attach ribs number five through seven and the upper abdomen subassembly. A lower thoracic spine tilt sensor and the upper abdomen assembly are mounted to this weldment. The top of the structure attaches to the upper spine flex joint and to the top of the lumbar pitch change mechanism.

Lumbar Pitch Change Mechanism - This new dummy posture adjustment system connects and is used for adjusting the angle between the lower thoracic and lumbar portions of the spine. Although the whole structure is modeled as a rigid body, these entities can be used to rotate the appropriate parts as in the physical dummy.

Lumbar Spine - This system, modeled as a rigid body, contains a five-axis thoracic spine load cell and has mounted to it a lumbar spine tilt sensor a triaxial accelerometer at the location of the twelfth thoracic vertebra (T12).
Lumbar Spine Flex Joint - This system provides flexibility between the lower spine and pelvic regions. Aside from being taller, it is constructed in the same manner as the upper spine flex joint. The lumbar spine flex joint attaches between the lumbar spine and pelvis-lumbar mounting subassemblies. The level of biofidelity of this flex joint is also not fully quantified due to the lack of dynamic biomechanical data.

The material and component tests performed are also identical to those for the upper spine flex joint. Figure 5 shows a component test where the top plate of the flex joint is attached to a stiff cylindrical bar that is impacted with another rigid bar with appropriate mass and initial velocity conditions.

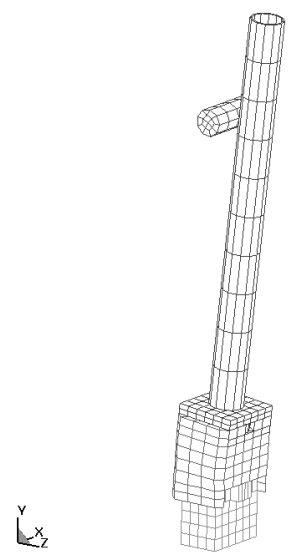

Figure 5. Lumbar Spine Flex Joint flexion test

The results of the validation of the flex joint urethane material model are shown in Figure 6.

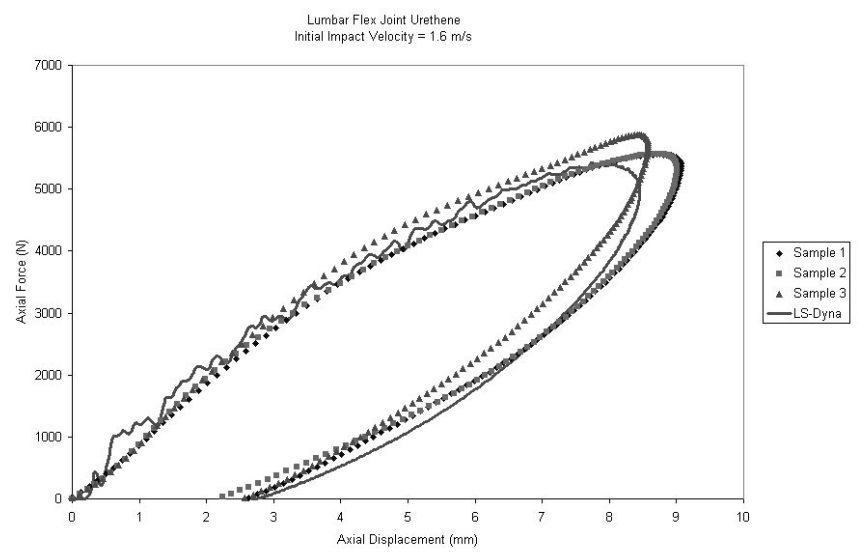

Figure 6. Flex Joint urethane material model validation at low speed

The graph in the figure shows the force-deflection response of the two-inch cube specimen to a $1.6 \mathrm{~m} / \mathrm{s}$ dynamic impact for the three physical tests as well as the LS-Dyna model. The same results are shown in Figure 7 at an impact speed of $2.8 \mathrm{~m} / \mathrm{s}$. The results at this higher speed indicate that the material model begins to deviate from the urethane behavior at higher impact velocities. 


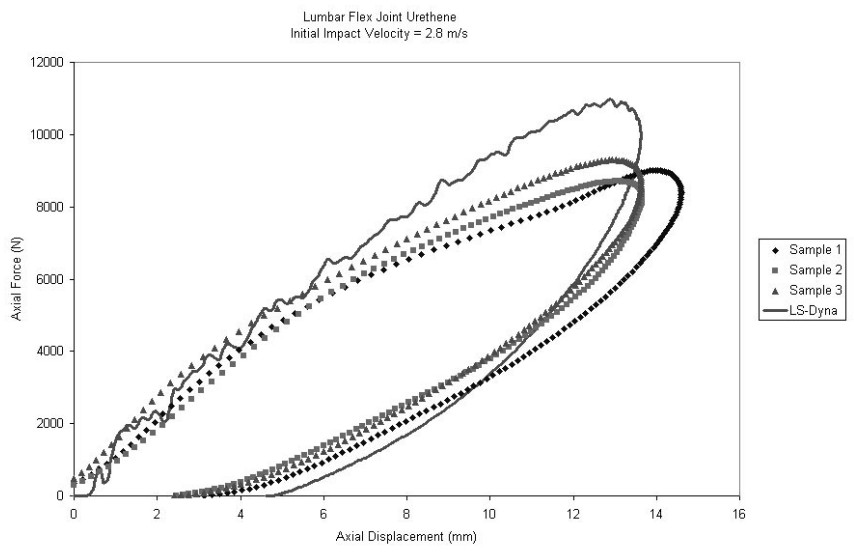

Figure 7. Urethane material model high-speed validation

The material model and corresponding parameters used to obtain these results were used in the frontal and lateral flexion validation of the flex joint component. The angular and moment time history responses of the low speed frontal impact are shown in Figures 8 and 9, respectively.

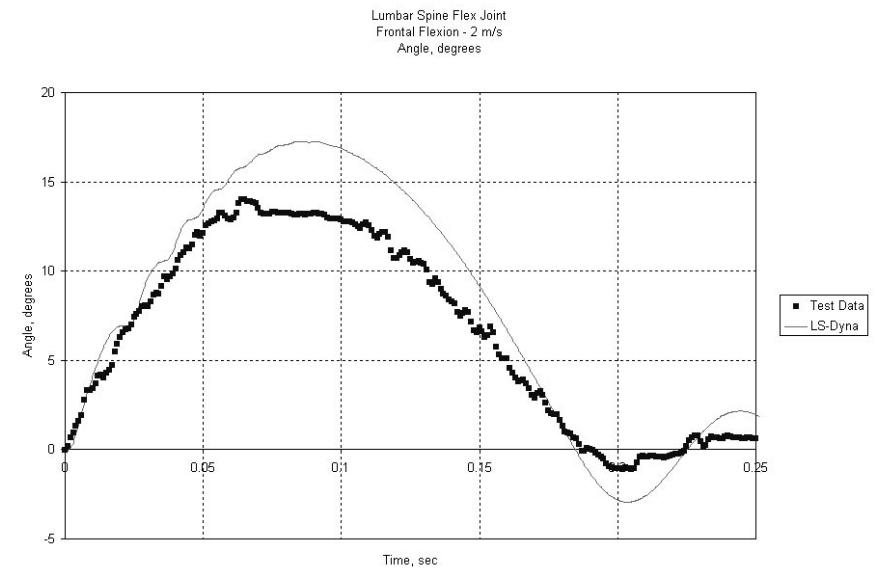

Figure 8. Low-speed frontal flexion angular response

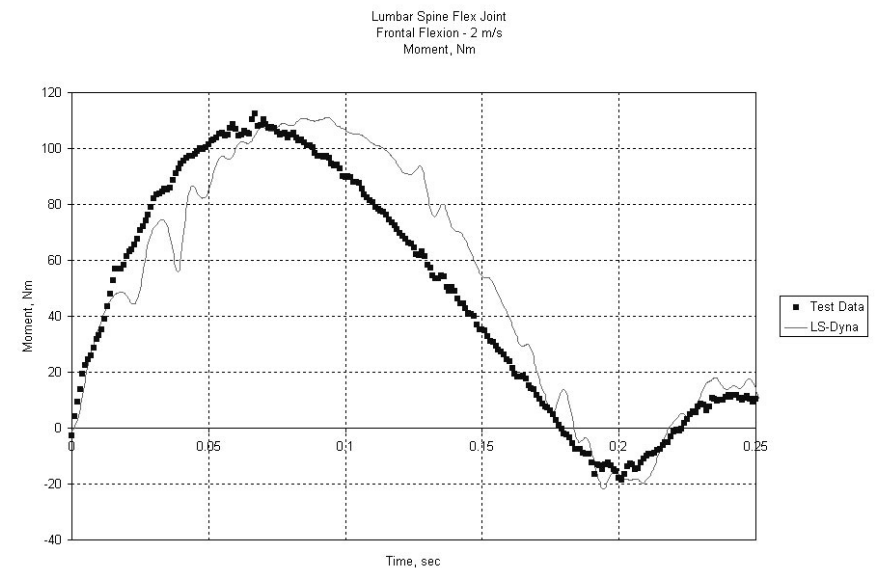

Figure 9. Low-speed frontal flexion moment response
The same results are shown in Figures 10 and 11 for lateral flexion.

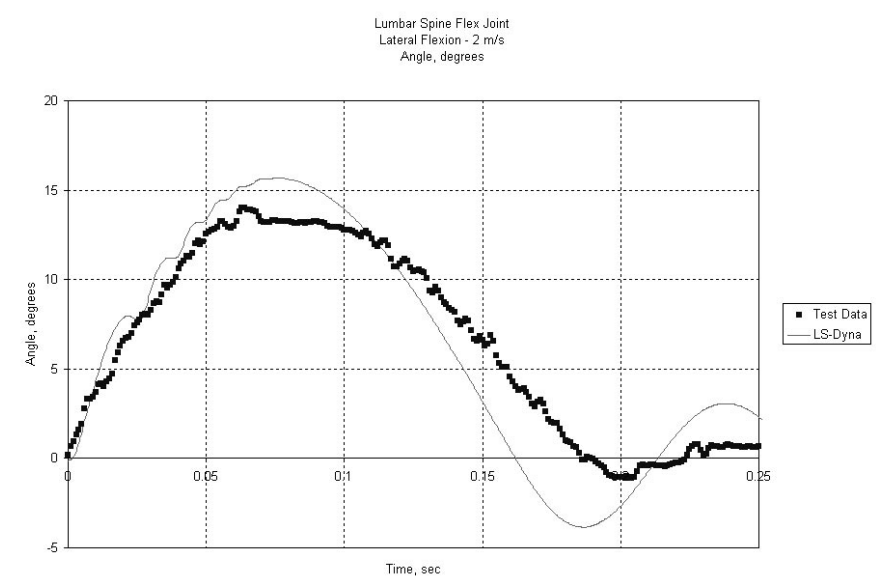

Figure 10. Low-speed lateral flexion angular response

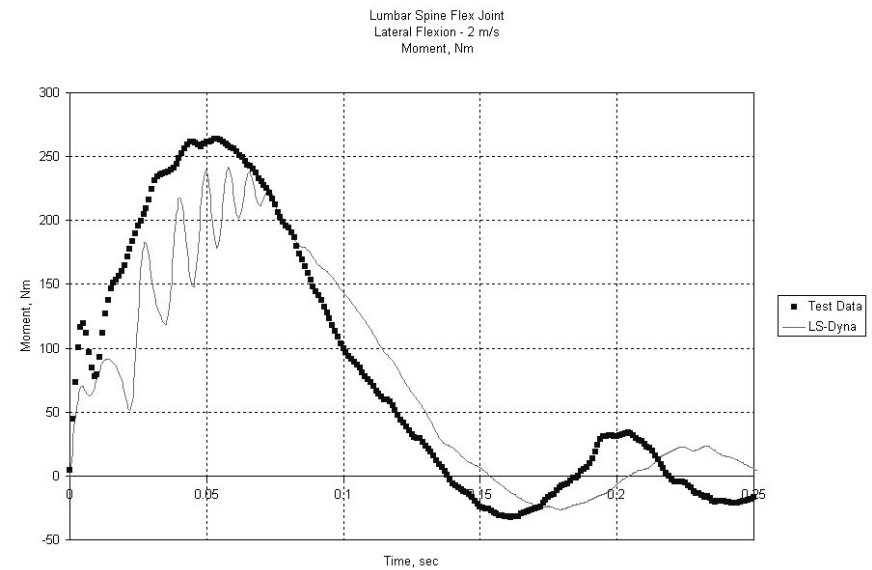

Figure 11. Low-speed lateral flexion moment response

Although not presented here, the correlation between the model and the test data begins to deviate when the flex joint is flexed at the higher impact velocity $(4 \mathrm{~m} / \mathrm{s})$ as seen in the material validation of the urethane.

Pelvis/Lumbar Mounting - This rigid part attaches the bottom of the lumber spine flex joint and to the pelvis casting, and contains a pelvic tilt sensor.

SHOULDERS - The shoulders are one of the major new design regions of the Thor dummy and consist of a series of linkages that connect the spine to the sternum. The shoulder connects to the upper thoracic spine via a shaft inserted in large shoulder blocks and to the upper arms via shoulder yokes. The shoulder supports rotate fore and aft with resistance controlled by wedge shaped Buna-N rubber blocks on each side that compress during shoulder rotation. The shoulder shaft contains a urethane bushing that allows for some vertical motion that corresponds to the human shrug motion. The support is connected via a resistance-free pin joint to the yoke. The yoke allows the arm to rotate about two independent 
axes and is also connected to the sternum via a clavicle. Each end of the clavicle has ball joints that allow some degree of rotation. The shoulder supports are each covered by a polyurethane shoulder pad.

Each component is modeled as described above with appropriate LS-DYNA joint entities in the yoke, clavicle, and support block. Figure 12 illustrates the finite element model of the shoulder assembly, where one shoulder pad is removed to show detail. The component tests performed on the subassembly include static fore and aft shoulder rotations to extract moment-rotation characteristics. Resistance to rotation of the clavicle ends was statically tested, as was the resistance of the shoulder block to vertical shrugging. The Buna-N rubber stops and polyurethane shoulder pad are modeled as viscoelastic. Compliance simulating the shoulder shrug motion is provided by a cylindrical joint and a series of spring elements around the bolt, passing through the urethane bushing in the shoulder block.

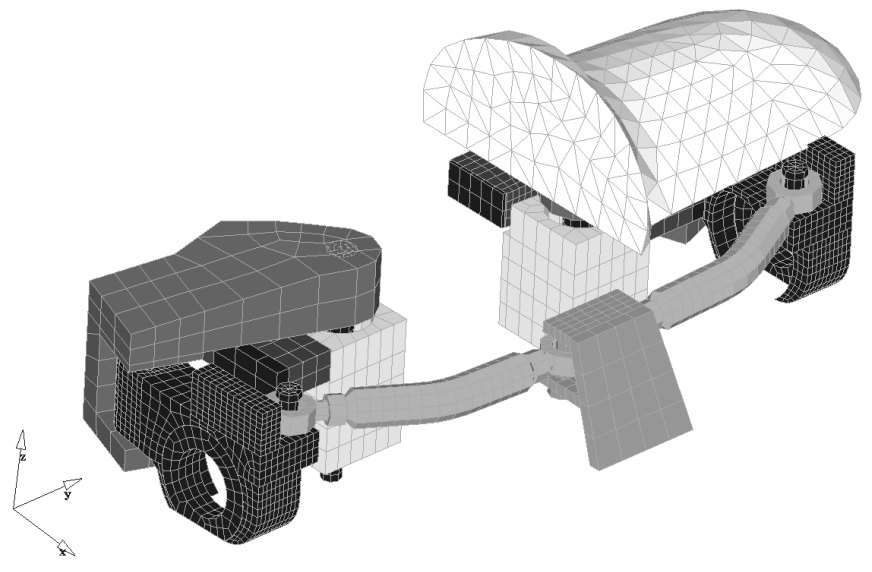

Figure 12. Shoulder assembly

MID-STERNUM - The mid-sternum consists of an ensolite damping pad and a sternum mass attached to a metal plate and is connected to the weighted bibs of the thorax subassembly. Figure 13 contains a view of the mid-sternum mesh as seen from the interior of the thorax. Each entity is modeled and the only deformable part is the damping pad. The mid-sternum is tested in conjunction with the thorax subassembly.

THORAX - The thorax is perhaps the most critical assembly in the Thor dummy and consequently, is modeled with considerable attention to detail. In addition to the shoulders and mid-sternum described above, the thorax consists of seven elliptically shaped ribs attached to the rear of the spine with two weighted bib layers and a urethane outer bib extending over the full thorax, over the shoulders, and down the back. To complete the assembly, the dummy torso is covered with a Rubatex jacket, which is still undergoing refinements. A short steel stiffener at the rib rear spine attachment stiffens each spring steel rib and a specially formulated rib damper is bonded on the rib interior to provide the correct force-deflection characteristics of an impacted ribcage. The ribs are vertically slanted and increase in cross-section from the top to bottom ribs, as seen in Figure 14, for more humanlike thorax anthropometry and rib contour. The ends of the ribs each connect to the bib layers and ribs three and six also have the CRUX units mounted on their left and right ends. The CRUX system, or compact rotary unit, is a new high-speed three-dimensional thorax deflection measurement system. There is also a five-axis thoracic load cell and a chest center-of-gravity accelerometer mounted in the spine.

All parts of the rib are modeled as deformable with the stiffeners and spring steel with elastic steel shells and the rib damper as viscoelastic bricks. The urethane bib is modeled with elastic shells, and the weighted bib layers with viscoelastic shells. A tension test was also performed on the urethane bib material to obtain its elastic properties.

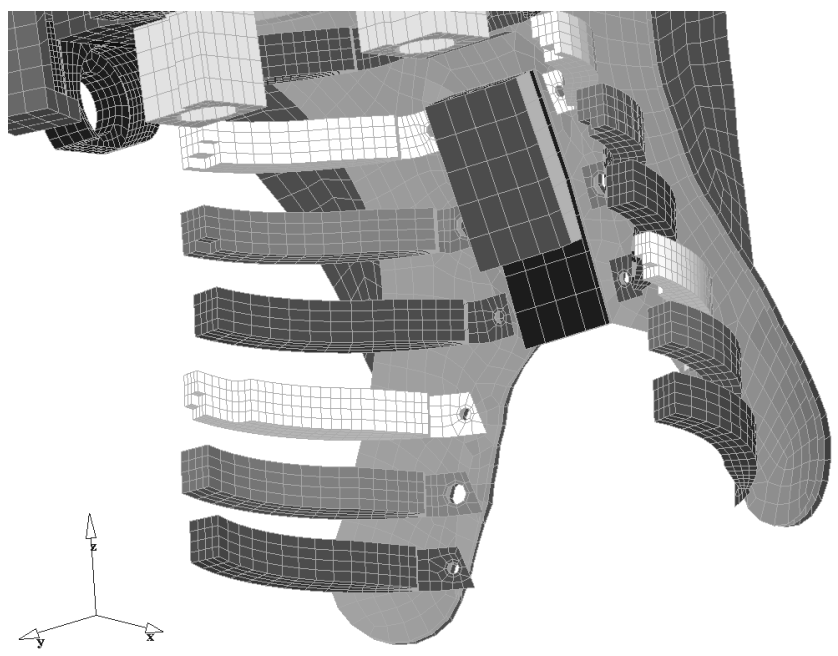

Figure 13. Mid-sternum in thoracic cavity

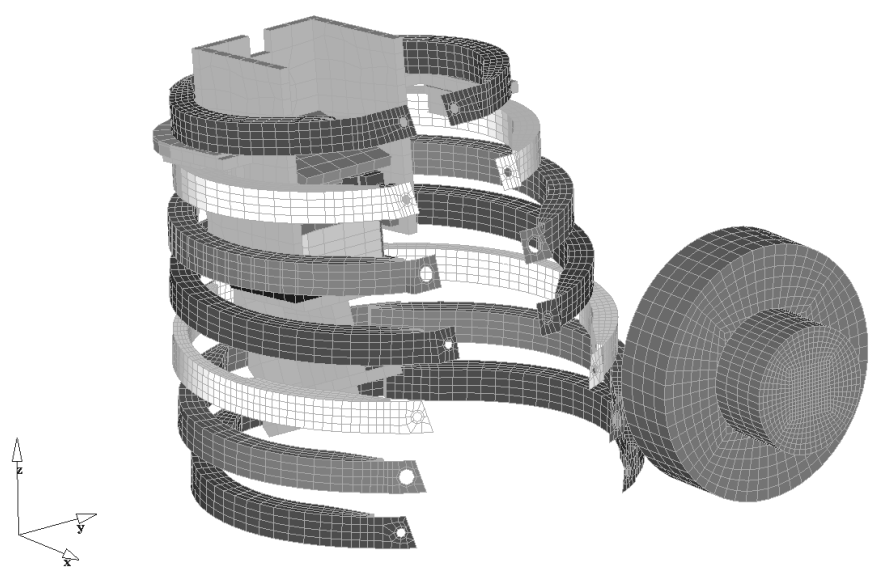

Figure 14. Ribcage assembly with impactor 
The method of fastening the ribs to the spine and bib layers is important. In general, fasteners that create a nonslip or rigid interface between components, are simulated by merging nodes on either side of the components. If the fasteners have significant mass, discrete masses are added. Also, although the dampers are bonded to the spring steel ribs, they are connected in the model by merging nodes at their common interface. This simulates a perfect bond.

A series of tests were performed on various parts of the ribcage to provide a graduated method for validating the finite element model. Each individual rib was dynamically impacted at two speeds horizontally, vertically angled at $15 x$, and horizontally angled at $30 x$. The normal horizontal testing was performed on the spring steel alone, the spring steel with only the damping material, and then all together with the stiffener. During the testing, the ends of the ribs were connected by a strip of bib material to prevent additional vibration. During all testing, especially with the temperature sensitive damping material, the temperature and humidity were recorded.

The full ribcage was then tested without any bibs, with only the outer urethane bib, and then all together with the 2 layers of weighted bib material. It should be noted that the impactor is wrapped with a piece of Rubayex jacket material to avoid the metal to metal contact with the fasteners on the rib ends. These tests were used to understand and model the contribution of each component in the ribcage in various dynamic impact conditions. These full ribcage tests also ensure that the internal contacts between components work properly

ABDOMEN - The new restorable abdomen design of the Thor dummy is broken up into two subassemblies. The Upper Abdomen lies between the bottom of the ribcage, and the Lower Abdomen is situated just below the ribcage in the pelvic cavity.

Upper - The upper abdomen subassembly essentially consists of foam blocks attached to the spine by a metal plate all enclosed in a fabric bag, as shown in Figure 15 (fabric bag in wireframe). A string potentiometer measures the centerline deflection at the outer surface of the upper abdomen and a single axis accelerometer is mounted there as well. A plastic load distribution plate distributes the load at the surface of the outer foam layer. The bases of the four CRUX units are all attached to this assembly which is attached to the lower thoracic spine.

The deformable parts of the model include one outer foam block layer, three sponge rubber foam inner layers, and the enclosing fabric bag. The deflections at the potentiometer and CRUX units are measured by very low stiffness spring elements. The charcoal polyester foam and neoprene sponge rubber were each modeled with low-density foam material models and the fabric bag modeled with elastic shells. Although the foam is compressed to fit in the bag upon dummy assembly, only the final geometry is modeled without pre-stressing the components. The component tests performed on the subassembly include a dynamic rod impact at two speeds.

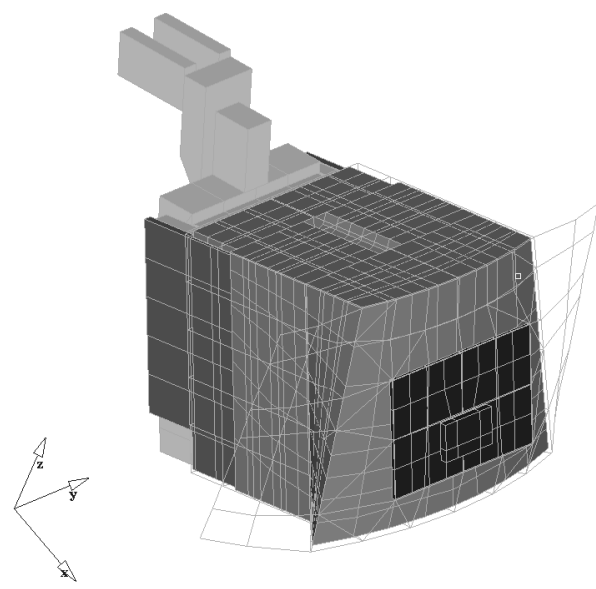

Figure 15. Upper abdomen subassembly

Lower - Similarly, the lower abdomen subassembly essentially consists of foam blocks supported to the spine by a metal plate all enclosed in a fabric bag, as shown in Figure 16. Two double gimballed string potentiometers (DGSP) measure the three dimensional penetration at the outer surface of the lower abdomen on the left and right sides. Plastic load distribution plates are also on each side where each DGSP attaches to the foam layer.

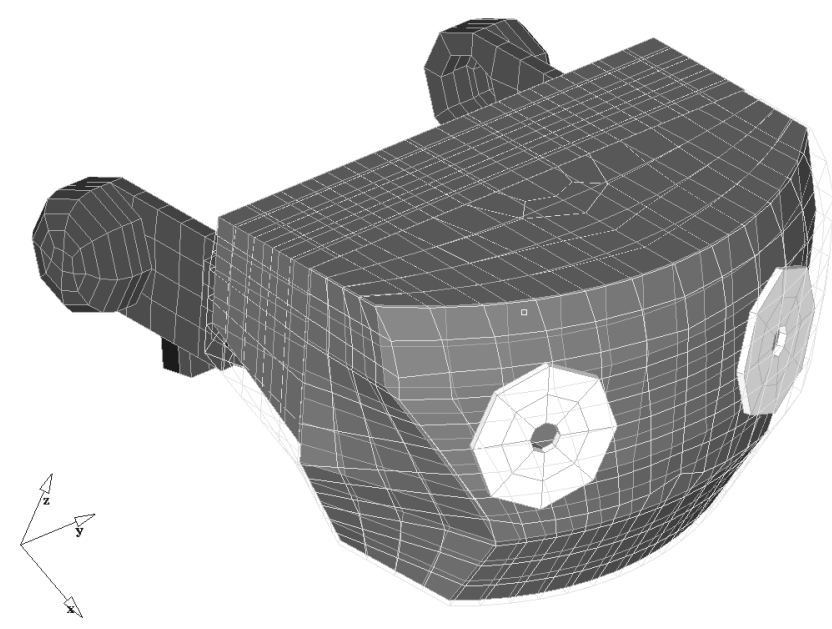

Figure 16. Lower abdomen subassembly

The model is essentially the same as for the upper abdomen. The deflections and rotations at the DGSPs are measured by very low stiffness springs. The materials used and component tests performed on the subassembly are the same as for the upper abdomen.

PELVIS - The Thor pelvis provides a more humanlike pelvic segmentation than existing dummies. The pelvis consists of a machined aluminum casting with left and right socket adaptors and Denton acetabular three-axis load cells. Figure 17 shows the pelvis subassembly 
model with the skin shifted to show the internal hardware. The socket adaptors house the pelvis-femur joint that is used to connect the leg to the pelvis. Also mounted on the pelvis is a triaxial accelerometer and pelvic tilt sensor. A molded silicone rubber skin encloses the pelvis casting. Only this skin is modeled as deformable and the static force-deflection characteristics are used since it will not experience high dynamic loading. Portions of the pelvis skin that are very thin are modeled with elastic shell elements instead of bricks with large aspect ratios.
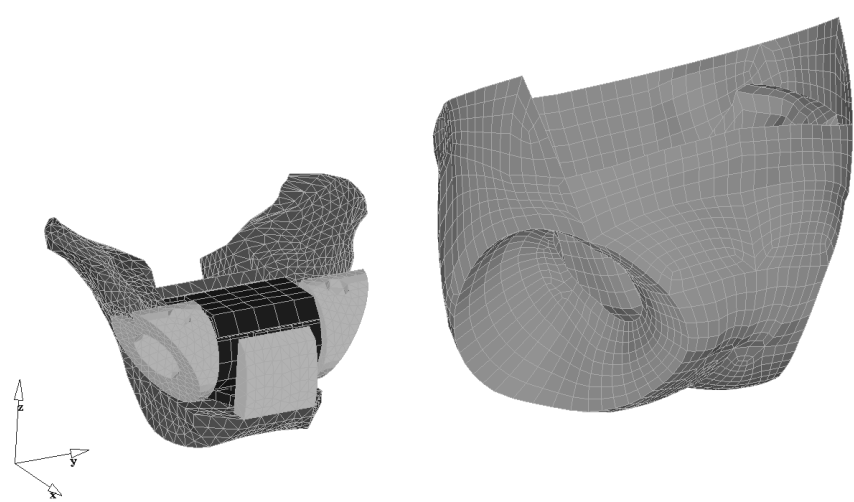

Figure 17. Pelvis subassembly

PELVIS/FEMUR JOINT - This joint merely consists of a metal casting from the Hybrid III dummy used to connect the femur to the pelvis. There is a friction adjustment set screw that adjusts the friction of the ball and socket joint in the pelvis attachment end. The joint is encased in a molded silicone rubber skin. The casting is modeled as a rigid body, the ball and socket joint as a spherical joint with friction, and the skin as an elastic material with static force-deflection characteristics.

FEMUR - The femur subassembly connects at one end to the pelvis/femur joint and to the knee structure at the other. The femur consists of a load cell in series with a shaft bearing housing, compliance bushing, sliding linear shaft, and shaft hub. The new compliant femur improves impact response. Each femur has a six-axis load cell. The shaft enforces only axial sliding of the compliant element. The femur skeleton described above is enclosed by a molded foam filled vinyl flesh covering.

The finite element model of the femur is shown in Figure 18. The only compliant parts in the structure are the neoprene rubber compliance bushing and femur skin, and were modeled as a viscoelastic material and a low density foam, respectively. The sliding shaft is modeled with a translational joint.

Component testing included axial and lateral disk impact tests at two speeds of the knee and femur structures together. Figure 19 shows the axial component simulation. The axial tests provide deflection effects from both the knee skin and compliance bushing and the lateral tests include the compliance of the femur flesh.

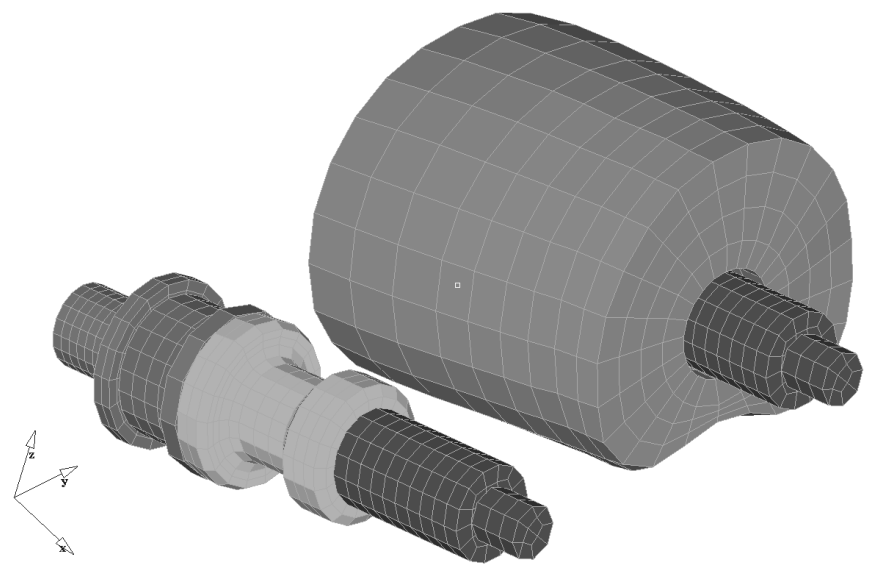

Figure 18. Femur subassembly

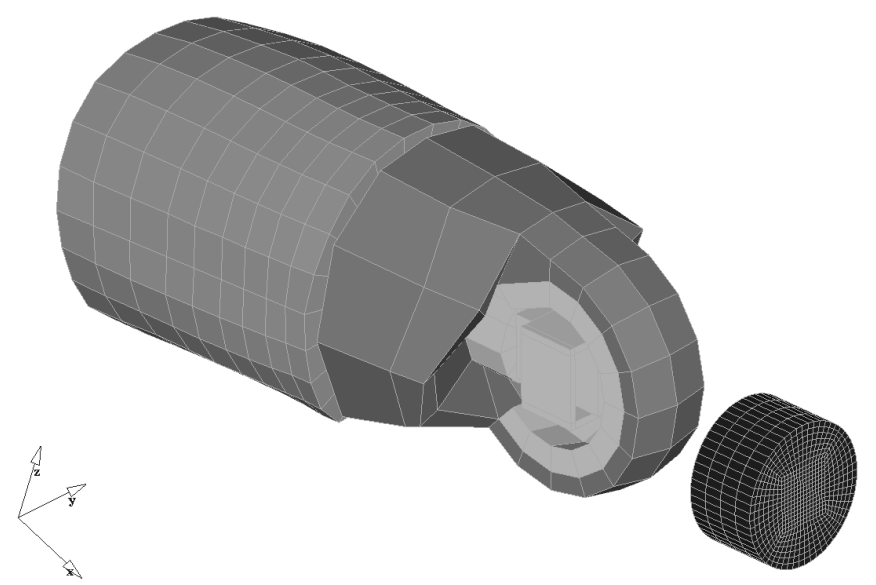

Figure 19. Knee/femur axial component impact simulation

KNEE - The knee component is taken from the Hybrid III dummy and connects the femur to the lower leg. It contains a metal casting, a pin joint, a knee sliding mechanism, and a foam filled vinyl skin covering.

The casting is modeled as a rigid body and the flesh is modeled the same as the femur flesh with a low density foam material. The component testing was described above with the femur.

LOWER LEGS AND FOOT/ANKLE - The lower leg and foot/ankle component is currently taken from the Hybrid III dummy and connects to the knee. It contains a metal skeleton and foam filled vinyl skin covering. A standard shoe from the Hybrid III dummy is also included.

The skeleton is modeled as a rigid body and the flesh is modeled the same as the femur flesh as a low density foam material. The finite element model of the lower legs was extracted from a public domain version of the Hybrid III dummy provided by LSTC [3] and is shown in Figure 20. The lower legs are currently being replaced with the newly designed Thor-Lx lower extremities. 


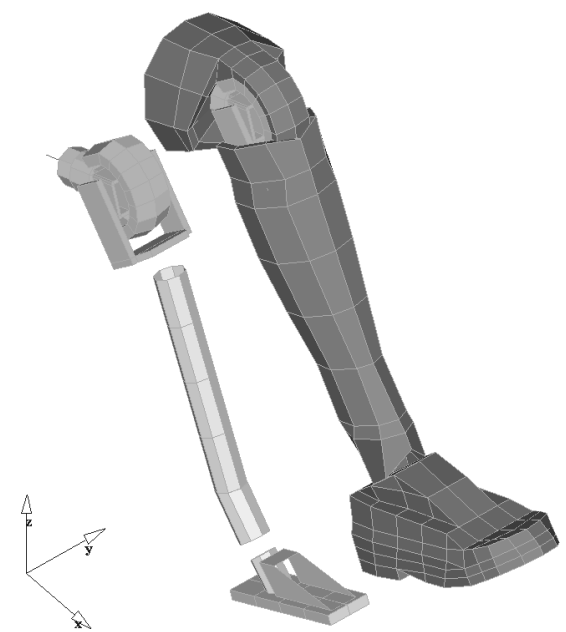

Figure 20. Lower leg and ankle/foot subassemblies

ARMS - The arm structure is also a rigid body skeleton with a low density foam flesh covering. Currently, the arms used on the Thor dummy are that of the Hybrid III dummy and are not described further here. The finite element model of the arms was also extracted from the LSTC model of the Hybrid III. Figure 21 contains the current finite element model of the arm assemblies. These entities will be developed further for a more accurate representation of the components.

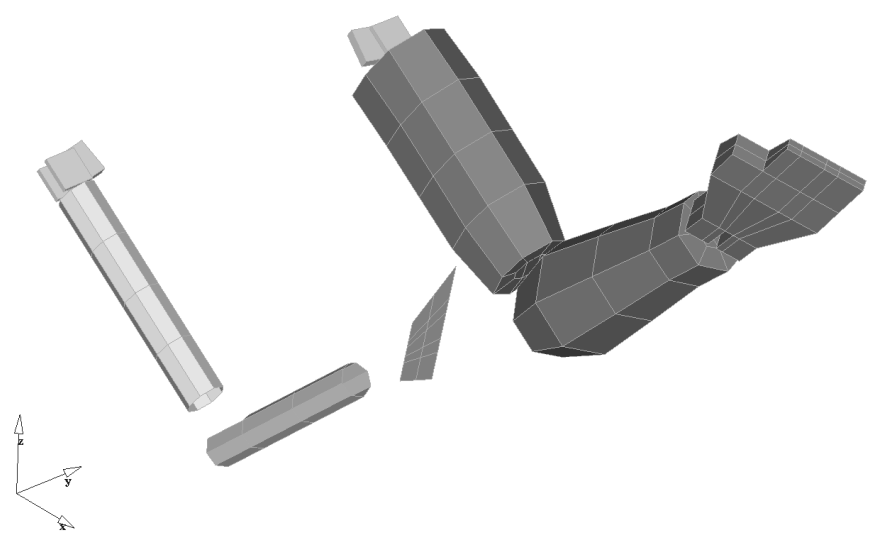

Figure 21. Arm assemblies

INSTRUMENTATION EQUIPMENT - The Thor dummy includes enhanced instrumentation such as dummy positioning sensors, acetabular load cells, and three-dimensional abdomen penetration measurement as well as other instrumentation used to quantify various response characteristics. Many of these instruments are easily represented in an LS-DYNA finite element model.

Deflections are measured in several locations either by string potentiometers, including a double gimballed string potentiometer (DGSP), or compact rotary unit (CRUX). The deflections measured by the CRUX units are easily represented by the relative displacement between two nodes within the model, so these instruments are modeled as nearly-zero-stiffness springs. The string potentiometers impose some stiffness so they are given an appropriately larger stiffness value.
Accelerometers throughout the dummy are represented within LS-DYNA as accelerometer elements that output accelerations in each local coordinate direction. The various load cells are modeled either as solid entities, stiff springs, or discrete beams where loads and moments can be extracted.

Also, there are various pitch change mechanisms to aid in positioning the physical dummy segments and rotary sensors to measure the rotation of certain segments. These are represented with nearly-zero-stiffness rotational spring elements to extract such information, including the rotations seen in the DGSPs.

MODEL STATUS - Although not yet validated at the full dummy level, the finite element model presented here consists of a total of 73,290 nodes and 59,030 elements divided among the subassemblies as described in Table 1. These do not include entities used for visualization purposes such as beam elements used to describe local coordinate system axes for instrumentation or joints.

\begin{tabular}{|l|c|c|c|c|}
\hline Assembly & $\begin{array}{c}\text { No. of } \\
\text { Parts }\end{array}$ & $\begin{array}{c}\text { No. of } \\
\text { Nodes }\end{array}$ & $\begin{array}{c}\text { No. } \\
\text { Deformable } \\
\text { Elements }\end{array}$ & $\begin{array}{c}\text { No. of } \\
\text { Rigid } \\
\text { Elements }\end{array}$ \\
\hline Head & 5 & 3009 & 700 & 1446 \\
\hline Neck & 5 & 5465 & 1460 & 2614 \\
\hline Spine & 16 & 4207 & 424 & 1430 \\
\hline Shoulder & 25 & 12050 & 2634 & 5959 \\
\hline Mid-Stern & 3 & 220 & 24 & 53 \\
\hline Ribs/Bibs & 25 & 18225 & 14454 & 0 \\
\hline Upper Ab & 10 & 2329 & 970 & 242 \\
\hline Lower Ab & 12 & 3740 & 1760 & 514 \\
\hline Pelvis & 14 & 8769 & 4788 & 9288 \\
\hline $\begin{array}{l}\text { Pelv/Fem } \\
\text { Joint }\end{array}$ & 4 & 2244 & 442 & 1268 \\
\hline Femur & 14 & 10870 & 1840 & 5558 \\
\hline Lwr Legs & 20 & 1556 & 496 & 260 \\
\hline Arms & 18 & 606 & 224 & 182 \\
\hline TOTAL & 171 & 7329 & 30216 & 28814 \\
\hline
\end{tabular}

Since modifications to the physical dummy continue to be made and performance testing and criteria for some components are not completely finalized, certain model components are not complete at this time. Periodic updates will be made to finalize the finite element model and to incorporate any significant future changes.

The most significant change to the Thor dummy is the addition of the newly designed lower extremities, Thor-Lx. Initially, the Thor dummy used the arms, knees, and lower legs from the existing Hybrid III crash test dummy and the model presented here reflects these components. The 
new Thor-Lx lower extremities are currently being incorporated into the Thor dummy model. Other areas not completely finalized include: neck cabling configuration, shoulder pad geometry, and rib damping material thickness. With these exceptions, the model presented herein contains the latest design of all Thor components. Details of the finite element model are illustrated in Figure 22 where some flesh/skin components have been removed to reveal underlying hardware.
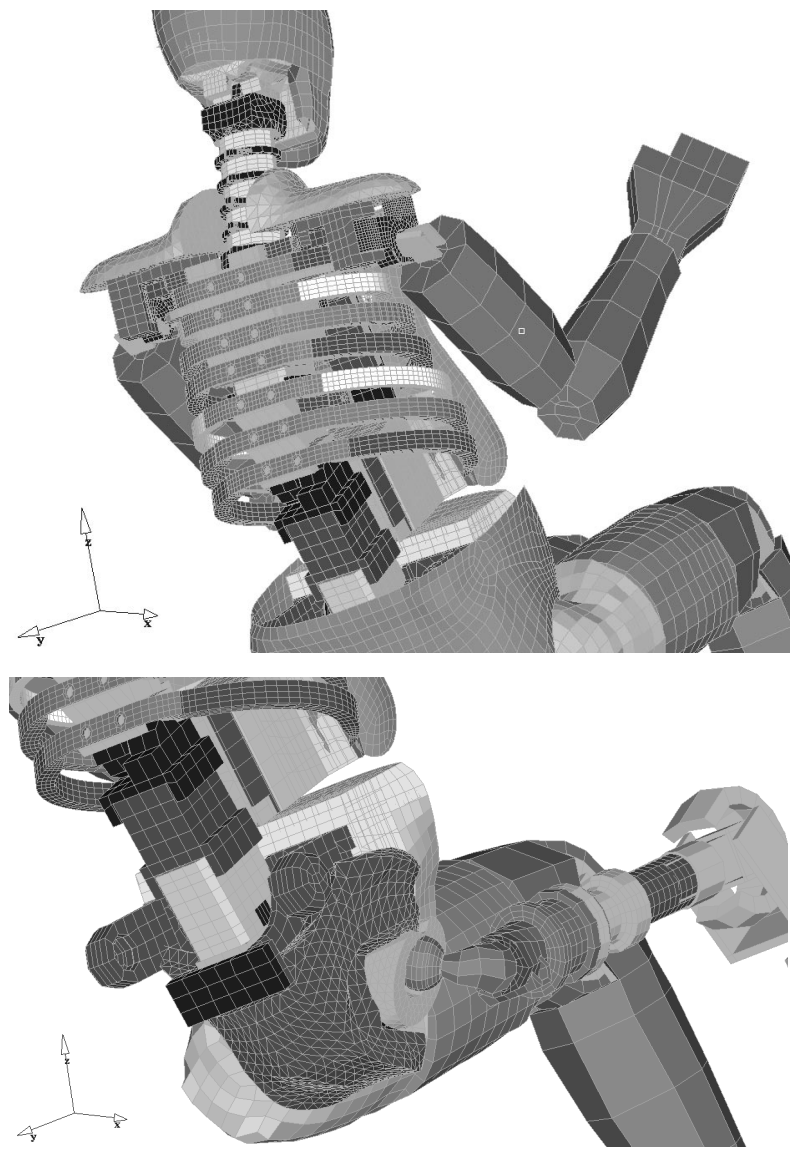

Figure 22. Model details

The fully validated components will be reassembled into one database and several tests performed to evaluate the full model performance.

The standard Kroell tests and corresponding simulations will be performed where a $23.4 \mathrm{~kg}$ mass is launched at $4.3 \mathrm{~m} / \mathrm{s}$ and $6.7 \mathrm{~m} / \mathrm{s}$ into the chest of the seated dummy, as shown in Figure 23. This test, also performed on cadavers, is used to develop the force-deflection corridor to evaluate the performance of the physical crash test dummy. Such a test also demonstrates how well all the dummy model components interact during a full impact. The main output correlated between the model and physical dummy includes the head, chest, and pelvic accelerations as well as the chest deflections at the CRUX units.

Dynamic abdomen and knee impacts like the ones described in the component testing will also be performed on the full seated dummy to check its performance under various impacts usually encountered in an automobile crash.
Whole-body head impacts are another method that may be used to evaluate how robust the complete model is. For each full dummy model test, the kinematics of the dummy will be investigated in addition to the instrumentation output throughout the dummy.

Belted sled tests and occupant compartment testing with an airbag and possibly a knee bolster are the most comprehensive crash environment for the finite element model to encounter. How the seat belts and airbag interact with the various components of the dummy model are essential to the usefulness of the finite element dummy model and these simulations will conclude the validation process.

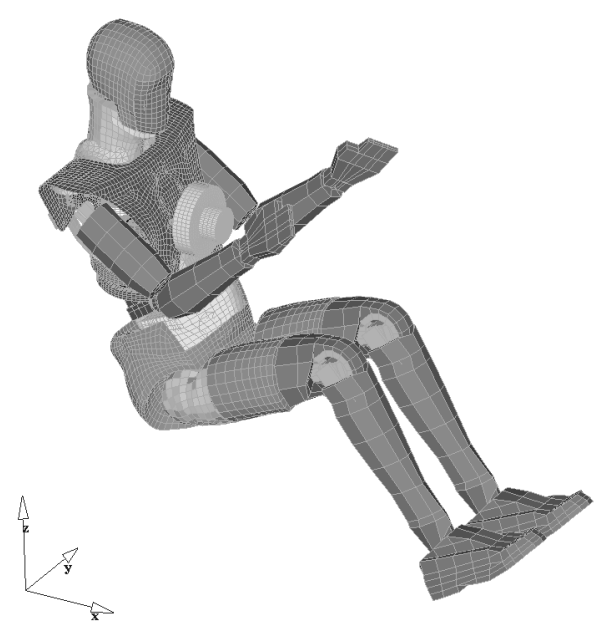

Figure 23. Kroell chest impact simulation

\section{CONCLUSION}

The procedure for developing a detailed finite element model of the new advanced crash test dummy, Thor, was presented. Material, subassembly, and full dummy validation testing and accompanying simulations were described that are used to create and validate the model of the recently developed Thor fiftieth percentile male dummy. The finite element modeling approach presented here can be used as a design tool for the expanding family of Thor crash test dummies, including the Thor fifth percentile female. The resulting model can also be an important crash safety tool useful for automotive safety systems design and development.

\section{REFERENCES}

1. GESAC, Inc., "Test Support for Finite Element Modeling of Thor Crash Test Dummy", May 1999 Volpe Center report.

2. Martinez, Luis and Garcia, Miguel, "Thor Sled Tests In European Seat Belt Restraint Conditions And Component Tests", Instituto Universitario de Investigacion del Automovil, July 1998

3. Livermore Software Technology Corporation, deformable Hybrid III 50th percentile male dummy model. 


\section{CONTACT}

For any information on the Thor dummy, contact:

Mark Haffner

U.S. Department of Transportaion

NHTSA National Transportation Biomechanics Research

Center

Washington, D.C. 20590

(202) 366-4719

mhaffner@nhtsa.dot.gov

Joseph Canha

U.S. Department of Transportation

RSPA/Volpe Center

Cambridge, MA 02142

(617) 494-2554

canha@volpe.dot.gov

Nagarajan Rangarajan, Tariq Shams

GESAC, Inc.

125 Orchard Drive

Boonsboro, MD 21713

gesac@erols.com 